\title{
HD gas purification for polarized HDice target production at Jefferson Lab
}

\section{C.S. Whisnant ${ }^{* a}$, A. D’Angelo ${ }^{b}$, L. Colaneri ${ }^{b}$, J.H. Devilbiss ${ }^{a}$, T. Kageya ${ }^{c}$, D. A. Loving $^{a}$, M. Lowry ${ }^{c}$, A. Rizzo ${ }^{b}$, A.M. Sandorfi ${ }^{c}$, C. Schaerf ${ }^{b}$, E. Speiser ${ }^{d}$, J.D. Storey ${ }^{a}$, C.M. Wallace ${ }^{a}, \mathbf{X}$. Wei $^{c}$, and I. Zonta ${ }^{b}$}

a James Madison University 901 Carrier Drive, Harrisonburg, VA

${ }^{b}$ Università di Roma Tor Vergata and INFN Roma Tor Vergata Via della Ricerca Scientifica, 100133 Rome, Italy

${ }^{c}$ Thomas Jefferson National Accelerator Facility 12000 Jefferson Avernue, Newport News, VA, USA

${ }^{d}$ Leibniz-Institut für Analytische Wissenschaften - ISAS - e.V., Department Berlin Albert-Einstein-Str.9, 12489 Berlin, Germany

E-mail: whisnacsejmu.edu, annalisa.dangelo@roma2.infn.it, luca.colanerieroma2.infn.it, hauverjsedukes.jmu.edu, kageya@jlab.org, lovin2daddukes.jmu. edu, mlowry@jlab.org, alessandro.rizzodroma2.infn.it, sandorfiejlab.org, carlo.schaerf@roma2.infn.it, eugen.speiser@isas.de, storeyjdedukes.jmu.edu, wallaccmedukes.jmu.edu, xwei@jlab.org, irene.zonta@roma2.infn.it

Frozen spin $H D$ targets have been used in the study of photonuclear reactions at Brookhaven National Laboratory and, more recently, at Jefferson Lab. Commercially available gas contains $H_{2}$ and $D_{2}$ at levels much too high for target production. To obtain gas the required purity, low temperature distillation is employed. The relative concentrations of $H_{2}$ and $D_{2}$ in the purified $H D$ gas are measured with a residual gas analyzer, gas chromatography, and Raman scattering. The distillation process is described and the measurement techniques and the results are summarized.

XVth International Workshop on Polarized Sources, Targets, and Polarimetry

September 9 - 13, 2013

Charlottesville, Virginia, USA

* Speaker. 


\section{Introduction}

Solid, frozen-spin targets of molecular $H D$, first developed for nuclear physics by a collaboration between Syracuse University and Brookhaven National Laboratory are now in use at Jefferson Laboratory. They have been successfully used in measurements with photon beams, first at the Laser-Electron-Gamma-Source [1] and most recently at Jefferson Lab during the running of the E06-101 (g14) experiment [2]. Preparations are underway to utilize the targets in future electron experiments after the completion of the $12 \mathrm{GeV}$ JLab upgrade $[3,4,5]$. HD is an attractive target since all of the material is polarizable, of low $\mathrm{Z}$, and requires only modest holding fields. At the same time, the small contributions from the target cell can be subtracted from direct measurements.

The polarization of $H$ in the $H D$ target is obtained by relaxation in a high magnetic field at low temperature. Achieving significant $D$ polarization is more complicated and includes rf transitions as well. The first rotational states of these molecules act as catalysts in polarizing $H D$ and small amounts are required to effect polarization. However, the decay of these excited molecular states also generates heat, limiting $H D$ polarization. This requires careful control of the $\mathrm{H}_{2}$ and $D_{2}$ concentration to obtain optimal polarizations. Commercially available $H D$ contains $0.5-2 \%$ $\mathrm{H}_{2}$ and $\mathrm{D}_{2}$, too high for target production. Low-temperature distillation is required to reduce these concentrations to the $\leq 10^{-4}$ to enable useful target production.

\section{Low Temperature Distillation}

All contaminants heavier than $\mathrm{He}$ are removed by cold traps and since $\mathrm{He}$ is not involved in the production process, only modest amounts of $\mathrm{H}_{2}$ and $D_{2}$ remain in the commercial $H D$ gas. Because the atomic ionization energy is much larger than the molecular disassociation energy, ionizing techniques are problematic. Distillation rather capitalizes on the significant mass dependent vapor pressure differences at low temperature.

Gas is distilled in batches of 12 moles. After condensation into the distillery at $10 \mathrm{~K}$, the system is closed and set to $18 \mathrm{~K}$ to liquify the $H D$. A heater boils the liquid creating a reflux through the column. Once a stable separation is obtained, the gas is extracted in a controlled manner. This entire process will take approximately three weeks.

Because the vapor pressure differences are on the order of 10-15\% even at temperatures between the triple point and the boiling point, a packed distillation column is used to enhance the separation. The column, approximately $5 \mathrm{~cm}$ in diameter, is filled with the commercial packing material, heli-pak [6] to increase its effective surface area. This permits repeated condensations and evaporations, each contributing another enhancement of $\approx 10 \%$ to the isotopic separation resulting in approximately an order of magnitude enhancement in the concentration.

The distillery is attached to the cold plate of a refrigeration system consisting of a two stage Joule-Thompson cooling loop using high purity $\mathrm{He}$ as the working gas. The first stage is cooled to $37 \mathrm{~K}$. To this stage are attached $A l$ heat shields that surround the distillation column, limiting the radiation heat load. The second stage reaches a base temperature of $10 \mathrm{~K}$. Attached to the second stage is the distillery consisting of two parts as shown in figure 1. The stainless steel upper portion contains a tube extending from the refrigerator cold plate through the full length of this portion. This extended cold finger liquifies $H D$ vapor rising from below. It contains liquid $H_{2}$ to stabilize 


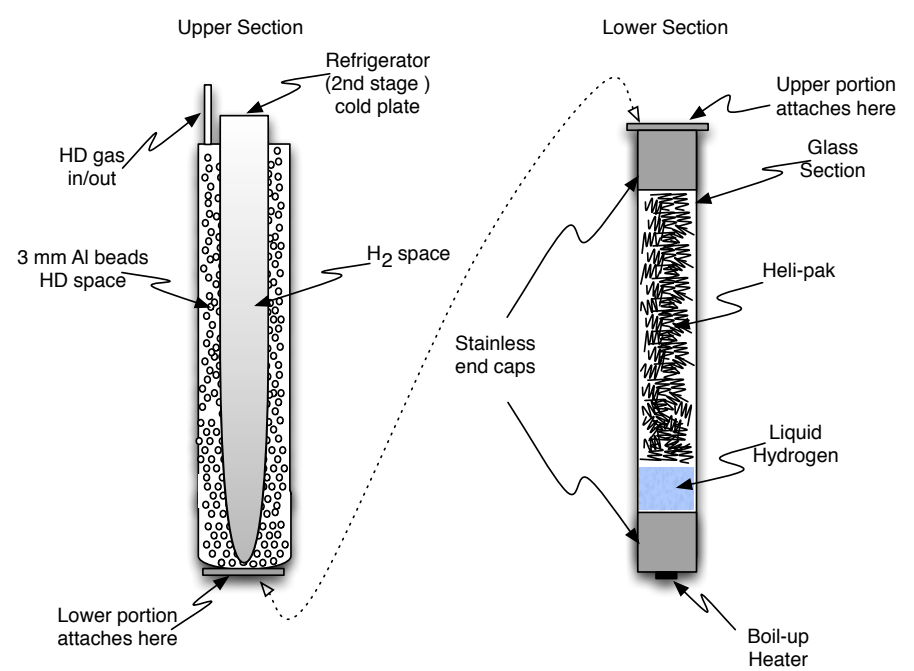

Figure 1: A schematic of the distillery. The upper portion is shown on the left. The packed column is illustrated on the right. The liquid hydrogen isomer mixture is boiled at the bottom of the lower portion of the column.

the temperature of this section. The condensation of the $H D$ on the outside of the cold finger boils the $\mathrm{H}_{2}$ on the inside which is liquified by the refrigerator. Using the latent enthalpy of the $\mathrm{H}_{2}$ in this way limits the temperature gradient along the length of the cold finger. To limit the total $H D$ volume of this section, it is filled with $A l$ beads $3 \mathrm{~mm}$ in diameter. This permits the free flow of gas and liquid while both filling space and providing more cold surface on which the boil-up can condense.

Below this stainless steel section is the glass distillation column. With viewing slots in the $A l$ heat shield and windows in the vacuum container, it possible to directly observe the state of the system. This permits viewing the liquid hydrogen to insure, for example, that the boil-up heater power is not set too high. The glass column contains the heli-pak that is the heart of the distillery. The boil-up heater is located at the bottom of the column.

Once the gas is condensed into the system, the system is closed and the temperature of the second stage is set to $18 \mathrm{~K}$. At this temperature, the pressure in the still is approximately $280 \mathrm{mbar}$.

Setting the boil-up heater power as high as possible is desirable. This allows the extraction of gas to be a minor perturbation on the reflux in the distillery. At a boil-up heater power near $2.3 \mathrm{~W}$ this maximum is reached and hydrogen gas moves upward through the column, condensing on the heli-pak and re-evaporating, no faster than the liquid hydrogen flows back down from the upper portion. This reflux reaches a steady state in a couple days. This results in a stratification with the lighter molecules higher in the column. Thus, at the start of extraction, the $\mathrm{H}_{2}$ is concentrated at the top of the distillery and the $D_{2}$ remains in the liquid at the bottom. The extraction rate is controlled using a mass flow controller calibrated for $\mathrm{H}_{2}$. Gas is extracted at a rate of $1 \mathrm{~mole} /$ day into a series of stainless steel tanks that each hold 2 moles at 1 ATM. At the distillery pressure of 280 mbar, each tank will contain approximately $\frac{1}{2}$ mole.

The composition of the extracted gas is initially monitored using a residual gas analyzer (RGA). Because this is an ionizing device, pure $H D$ gas results in $\approx 2 \% \mathrm{H}_{2}$ due to disassocia- 
tion/recombination in the RGA. This effectively limits the minimum $\mathrm{H}_{2}$ observable concentration and the range of usefulness of this method. At the beginning of extraction, the packed column enhances the concentration of $H_{2}$ at the top of the column to $15-18 \%$. This $H_{2}$ enriched gas is extracted into storage and monitored with the RGA. This is stage of the distillation shown in the left part of figure 2 .

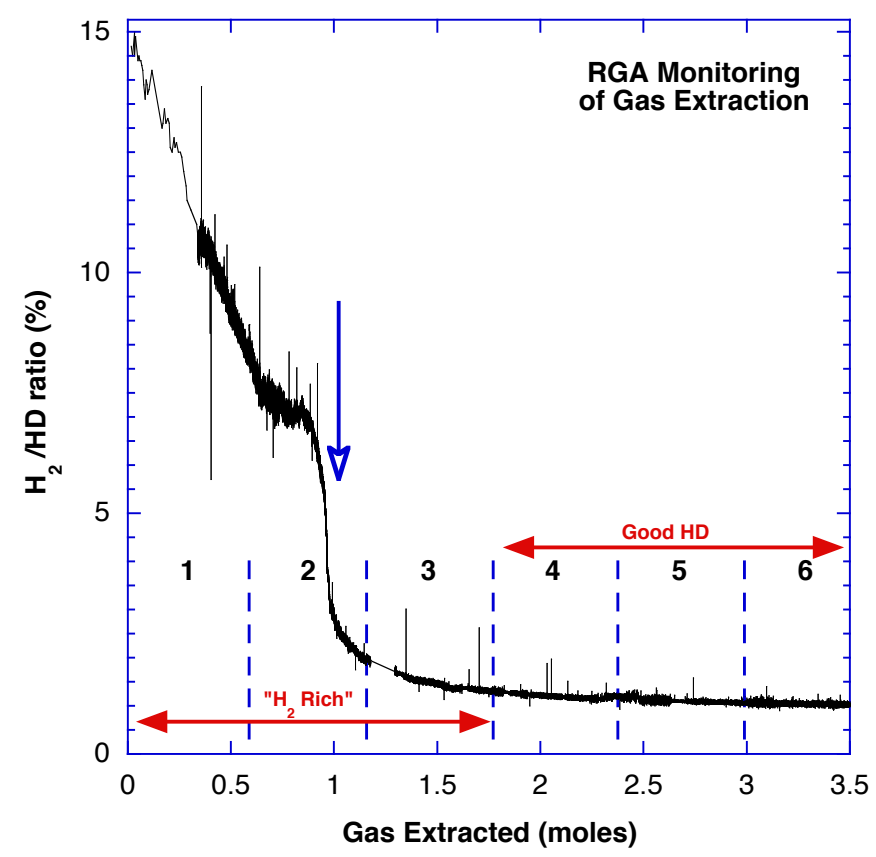

Figure 2: The RGA output for gas extracted from the distillery. By the $4^{\text {th }}$ tank, the RGA has reached its minimum $\mathrm{H}_{2}$ output independent of the actual $\mathrm{H}_{2}$ concentration. The vertical dashed lines indicate the storage tank changes. The extraction of three tanks ( 1.5 moles) effectively removes all the $H_{2}$ and the response is at its minimum value. The arrow indicated the extraction of all $\mathrm{H}_{2}$ estimated from the bulk content of the raw gas.

As seen in the figure, the $\mathrm{H}_{2}$ concentration falls from the beginning of the run and there is an abrupt drop while extracting gas into the second tank. This drop corresponds to the removal of the $H_{2}$ as calculated from the bulk content. To be conservative, a third tank is extracted to insure that all possible $\mathrm{H}_{2}$ is removed. Beginning with the fourth tank, purified $H D$ is stored.

Extraction continues until all liquid hydrogen is gone. This leaves 3.5 moles in the upper portion of the distillery. This is more than enough to insure that no $D_{2}$ is extracted so that purified $H D$ is obtained until the end of of the run. Extracting 1.5 moles of $H_{2}$ rich gas and leaving 3.5 moles of $D_{2}$ rich gas in the distillery yields of 7 moles of purified $H D$ per run.

To verify the content of the gas beginning with the third and fourth tanks, a more sensitive method is required. Gas chromatography provides a simple, non-ionizing method for greater sensitivity and this is described in the next section.

\section{Gas Chromatography}

Using gas chromatography to measure the $H_{2}$ and $D_{2}$ content of the distilled gas is described 
in detail in Whisnant, et al. [7] and is only briefly summarized here.

It is possible to create a chromatogram with good separation of $H_{2}, H D$, and $D_{2}$ using an offthe-shelf chromatography system. The column is a $30 \mathrm{~m}$ ValcoPLOT VP-Molesieve with an ID of $0.53 \mathrm{~mm}$ and film thickness of $50 \mu \mathrm{m}$. the carrier gas is high purity $N e$ and the signal is generated by the differential output of two thermal conductivity detectors. The column is immersed in a mixture of $L N_{2}$ and iso-pentane to hold the column at a temperature between 115 and $130 \mathrm{~K}$. The chromatogram is the product of the amount of each species of gas and their thermal conductivity. After correction for the thermal conductivities, relative concentrations are computed from the integrated peak areas. The chromatogram for gas from the fourth tank in the run shown in figure 2, is compared to the raw gas in figure 3 . There are four peaks in the chromatogram since there are separate peaks for ortho- and para- $\mathrm{H}_{2}$. This arises from a spin dependence in the interaction between the $\mathrm{H}_{2}$ and the proprietary stationary phase.

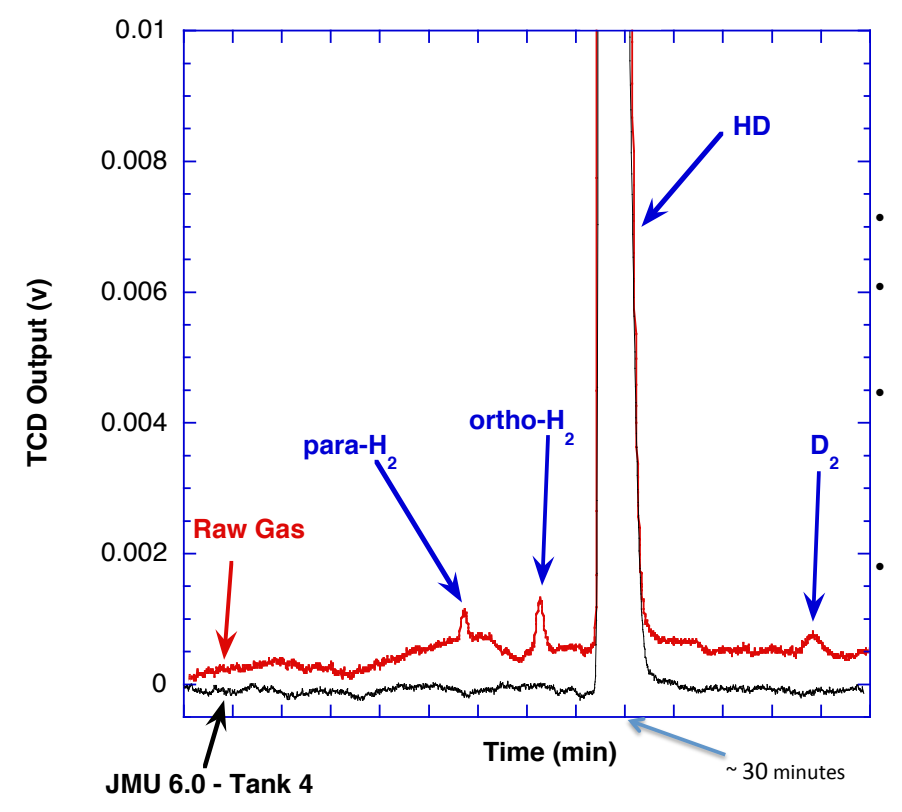

Figure 3: A comparison of the chromatogram of the raw, commercial gas and the gas in the fourth tank of the distillery run shown above. Note the absence of the both the $H_{2}$ and $D_{2}$ peaks in the distilled gas. The horizontal scale is approximate since small shifts are required to align the $H D$ peaks for comparison since the column temperatures are slightly different .

Gas chromatography has a sensitivity of a few parts in $10^{-3}$. This is sufficient to verify that the distillery is working correctly but to make a quantitative assay, a more sensitive technique is required.

\section{Raman Scattering}

The inelastic scattering of light from molecular hydrogen is a non-ionizing method for gas assay that provides even greater sensitivity than gas chromatography. The application of this spectroscopic method to the analysis of hydrogen gas is described in detail elsewhere [8]. 


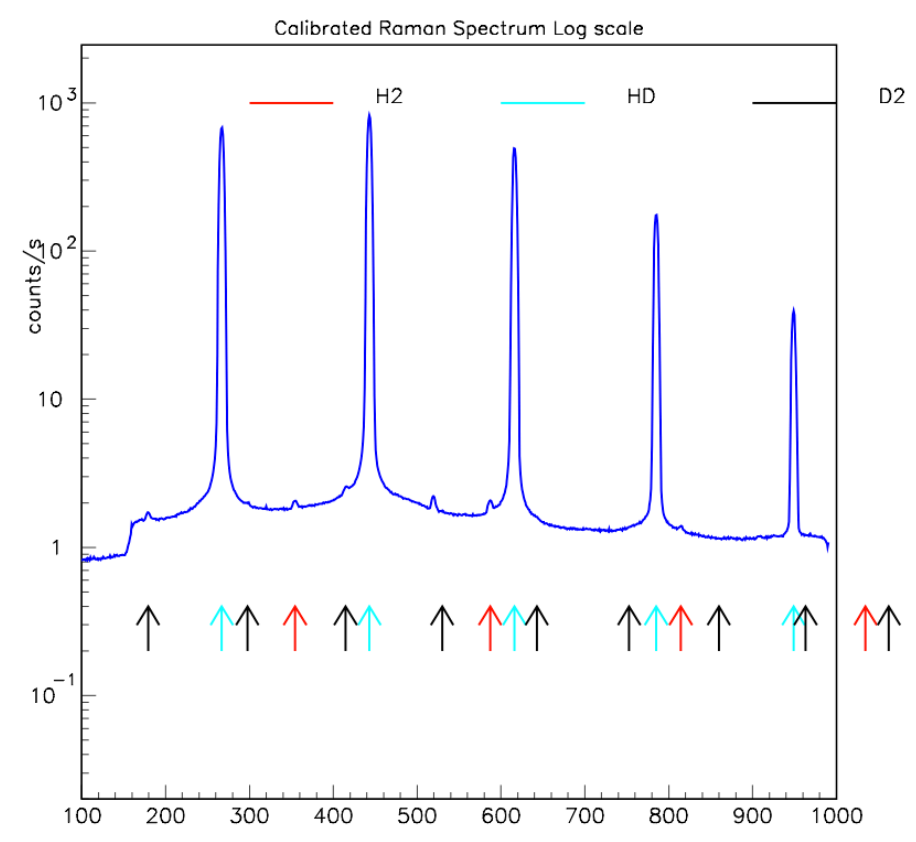

Figure 4: The Raman spectrum from distilled gas. The units on the horizontal axis is $\mathrm{cm}^{-1}$. For this sample, $H_{2} / H D=(20 \pm 5) \times 10^{-5}$ and $D_{2} / H D=(13 \pm 12) \times 10^{-5}$. For this sample, no $H_{2}$ or $D_{2}$ peaks were visible in the chromatogram. The colored arrows indicate the expected locations of the various $H_{2}, D_{2}$, and $H D$ lines.

The observed transitions are governed by the selection rule $\Delta J= \pm 2$ and connect ortho- to ortho- and para- to para-states in $H_{2}$ and $D_{2}$. For all three isomers there are a series of equally spaced transitions observed making possible the unambiguous quantification of each species even if a few lines cannot be resolved.

Figure 4 shows the Raman spectrum for gas taken from a recent distillation run. As indicated, there are many lines expected but few observed. These lines are fit with a Gaussian shape and areas extracted. Data are collected in the range from 100 to $1000 \mathrm{~cm}^{-1}$ higher than the original laser frequency (stokes lines). The ortho-ortho transitions and those involving lower angular momentum produce higher intensities making some transitions visible at small concentrations and others not. Combining this with the temperature dependence, it is possible to determine the relative concentrations of the isomers.

In the particular spectrum shown in figure 4, the extracted ratios are $H_{2} / H D=(20 \pm 5) \times 10^{-5}$ and $D_{2} / H D=(13 \pm 12) \times 10^{-5}$. This is well below the detection limits of gas chromatography and the chromatogram for this gas indeed shows no $\mathrm{H}_{2}$ or $\mathrm{D}_{2}$ peaks.

\section{Summary}

Gas chromatography and Raman scattering are found to be within experimental uncertainties in an analysis of raw, undistilled gas. The RGA, gas chromatography, and Raman scattering provide a series of consistent techniques of increase sensitivity that permit quantifying the results of gas 
distillation. With these methods, we have shown distillation to provide gas of sufficient purity for target production.

\section{References}

[1] S. Hoblit, et al., Measurements of $\vec{H} \vec{D}(\vec{\gamma}, \pi) X$ and the Convergence of the GDH Integral, Phys. Rev. Lett. 102 (2009) 172002.

[2] CLAS Collaboration, F. Klein and A.M. Sandorfi (spokesmen), $N^{*}$ Resonances in Pseudoscalar-meson photo-production from Polarized Neutrons in HD and a complete determination of the $\gamma n \rightarrow K_{0} \Lambda$ amplitude, www.jlab.org/exp_prog/proposals/06/PR06-101.pdf

[3] CLAS Collaboration, H. Avakian, S. Anefalos Pereira, A. Courtoy, M. Radici and K. Griffioen (spokespersons), Measurement of transversity with dihadron production in SIDIS with transversely polarized target, wWw.jlab.org/exp_prog/proposals/12/PR12-12-009.pdf

[4] CLAS Collaboration, H. Avakian, V.D. Burkert, L. Elouadrhiri, M. Lowry, M. Guidal and S. Procureur (spokespersons), Deeply Virtual Compton Scattering at $11 \mathrm{GeV}$ with transversely polarized target using the CLAS12 Detector, www.jlab.org/exp_prog/proposals/12/PR12-12-010.pdf

[5] CLAS Collaboration, H. Avakian, F. Klein, M. Aghasyan, K. Joo and M. Contalbrigo (spokespersons), Transverse spin effects in SIDIS at $11 \mathrm{GeV}$ with a transversely polarized target using the CLAS12 Detector, www.jlab.org/exp_prog/proposals/11/PR12-11-111.pdf

[6] T. Ohta, et al., Distillation of hydrogen isotopes for polarized HD targets, Nucl. Inst. Meth. 664 (2012) 347, www.wilmad-labglass.com/Products/LG-6730-104/

[7] C.S. Whisnant, et al., Measuring the relative concentration of $\mathrm{H}_{2}$ and $\mathrm{D}_{2}$ in $\mathrm{HD}$ gas with gas chromatography, Rev. Sci. Instrum. 82, (2011) 024101.

[8] A. D’Angelo, A. Fantini, C. Schaerf, V. Vegna, B. Buick, S. Del Gobbo, W. Richter, E. Speiser, A. Deur, T. Kageya, M. Lowry, A. Sandorfi, X. Wei, and C.S. Whisnant, HD Gas Distillation and Analysis for HD Frozen Spin Targets, Proceedings of the 13th International Workshop on Polarized Sources, Target and Polarimetry, Ferrara, Italy, 7-11 September 2009, pp 123-130, World Scientific Publishing Co. Pte. Ltd. 